\title{
ASSOCIAÇÃO ENTRE IMPULSIVIDADE E IDEAÇÃO SUICIDA EM ESTUDANTES UNIVERSITÁRIOS
}

\section{ASSOCIATION BETWEEN IMPULSIVITY AND SUICIDAL IDEATION AMONG COLLEGE STUDENTS}

\section{ASSOCIACIÓN ENTRE IMPULSIVIDAD E IDEACION SUICIDA EN ESTUDIANTES UNIVERSITARIOS}

\begin{abstract}
Hugo Gedeon Barros dos Santos ${ }^{1}$, Samira Reschetti Marcon ${ }^{2}$, Alice Milani Nespollo ${ }^{3}$, Jocilene de Carvalho Miraveti ${ }^{4}$, Moisés Kogien ${ }^{5}$, Eveline do Amor Divino ${ }^{6}$
\end{abstract}

Como citar esse artigo: Santos HGB, Marcon SR, Nespollo AM, Miraveti JC, Kogien M, Divino EA. Associação entre impulsividade e ideação suicida em estudantes universitários. Rev Enferm Atenção Saúde [Internet]. 2021 [acesso em___];10(2):e202114. doi:10.18554/reas.v10i2.4269

\section{RESUMO}

Objetivo: avaliar a associação entre impulsividade e ideação suicida em estudantes universitários. Método: estudo transversal com 567 estudantes de uma universidade pública. Utilizou-se a Escala de Impulsividade de Barratt para avaliação da impulsividade; questionário para caracterização sociodemográfica e aferição de ideação suicida nos últimos 30 dias. As associações foram verificadas pelo teste qui-quadrado de Pearson e exato de Fisher, com nível de significância de $5 \%$ e intervalo de confiança de $95 \%$. Utilizou-se o programa SPSS 17.0. Resultados: a prevalência de ideação suicida foi 9,7\%, estando mais presente entre mulheres e sujeitos entre 18 e 24 anos; 65,96\% encontravam-se dentro dos limites normais de impulsividade. Houve associação significativa entre impulsividade e ideação suicida, sendo os grupos de universitários "altamente impulsivos" e "muito controlados" os mais expostos. Conclusão: a maioria dos estudantes manteve-se dentro dos padrões normais de impulsividade, contudo os discentes altamente impulsivos e muito controlados apresentaram associação significativa com presença da ideação suicida.

Descritores: Suicídio; Ideação Suicida; Comportamento Impulsivo; Estudantes; Universidades.

\footnotetext{
${ }^{1}$ Enfermeiro, MSc, Unidade de Atenção Psicossocial, Hospital Universitário Julio Muller, Cuiabá, MT, Brasil.

${ }^{2}$ Enfermeira, PhD, Professora Adjunta, Faculdade de Enfermagem, Universidade Federal de Mato Grosso, Cuiabá, MT, Brasil.

${ }^{3}$ Enfermeira, Msc, Faculdade de Enfermagem, Universidade Federal de Mato Grosso, Cuiabá, MT, Brasil.

${ }^{4}$ Enfermeira, PhD, Professora Adjunta, Faculdade de Enfermagem, Universidade Federal de Mato Grosso, Cuiabá, MT, Brasil.

${ }^{5}$ Enfermeiro, Msc, Faculdade de Enfermagem, Universidade Federal de Mato Grosso, Cuiabá, MT, Brasil.

${ }^{6}$ Enfermeira, PhD, Professora Adjunta, Faculdade de Enfermagem, Universidade Federal de Mato Grosso, Cuiabá, MT, Brasil.
} 


\begin{abstract}
Objective: To evaluate the association between impulsivity and suicidal ideation among college students. Method: Cross-sectional study, participants included in the study were 567 students from a public university. The Barratt Impulsiveness Scale was used for impulsivity assessment and a questionnaire for sociodemographic description and measurement of suicidal ideation in the last 30 days. Possible associations were verified by the Pearson's chi-square test (or Fisher's Exact test, when appropriate), were adopted an interval of 95\% and significance level of 5\%. Results: The prevalence of suicidal ideation was $9.7 \%$, was more prevalent among women and subjects between 18-24 years old; as for the impulsivity level, 65,96\% of the participants are within normal limits. There was a significant association between impulsivity levels and suicidal ideation; students classified into "highly impulsive" or "extremely overcontrolled" groups were more likely to present suicidal thoughts. Conclusions: These findings corroborate the results of other studies and reinforce the need of expanding this topic discussion into universities and the implementation of preventive strategies.
\end{abstract}

Descriptors: Suicide; Suicidal Ideation; Impulsive Behavior, Students; Universities.

\title{
RESUMEN
}

Objetivos: Evaluar la asociación entre impulsividad e ideación suicida en estudiantes universitarios. Método: Estudio transversal con 567 estudiantes de una universidad pública. Se utilizó la Escala de Impulsividad de Barratt para evaluar la impulsividad y un cuestionario para caracterización sociodemográfica y evaluación de ideación suicida en los últimos 30 días. Las asociaciones se verificaron por medio de la prueba chi-cuadrado de Pearson y la prueba exacta de Fisher, se consideró un nivel de significación del 5\% y un intervalo de confianza del 95\%. Resultados: La prevalencia de ideación suicida fue de 9,7\%, predominó más en mujeres e individuos entre 18 y 24 años; el 65,96\% estaba dentro de los límites normales de impulsividad. Hubo una asociación significativa entre la impulsividad y la idea suicida, los grupos universitarios "altamente impulsivos" y "muy controlados" son los más expuestos. Conclusión: La mayoría de los estudiantes permaneció dentro de los patrones normales de impulsividad, sin embargo, los estudiantes altamente impulsivos y los muy controlados mostraron una asociación significativa con la presencia de ideación suicida.

Descriptores: Suicidio; Ideación Suicida; Comportamiento Impulsivo; Estudiantes; Universidades.

\section{INTRODUÇÃO}

O suicídio constitui-se na atualidade um grave problema de saúde pública que afeta, com diferentes níveis de intensidade, populações em todas as regiões do globo e de diferentes faixas etárias. ${ }^{1}$ Dados da Organização Mundial da Saúde (OMS) estimam que mais de 800 mil pessoas se suicidam anualmente, sendo a segunda causa de morte na população de 15 a 29 anos, precedida apenas pelas mortes causadas por acidentes de trânsito. ${ }^{2}$

Fenômeno complexo, o suicídio é o ato final de um problema multifacetado e multidimensional que engloba, ainda, outros componentes, como a ideação suicida e as tentativas de suicídio, constituindo o denominado comportamento suicida. ${ }^{3}$ A ideação suicida (ter diferentes níveis de pensamentos de retirar a própria 
vida, acompanhados ou não de planejamento) é um preditor significativo para o suicídio e/ou suas tentativas ${ }^{2-3}$, principalmente para a população de adolescentes e adultos jovens cuja prevalência de pensamentos de pôr fim à própria vida tem aumentado de maneira preocupante nos últimos anos. ${ }^{2,4}$

Entre essa população, evidências epidemiológicas têm apontado que alguns subgrupos, como os estudantes universitários, estão expostos a um maior risco para apresentar ideação suicida. ${ }^{5-8} \mathrm{O}$ ingresso em um curso superior, em geral, ocorre em uma fase da vida compreendida entre 18 e 24 anos, comumente marcada por mudanças sociais significativas. ${ }^{5}$ Nesse período, o estudante universitário, além de lidar com as novas demandas e pressões da vida adulta, deve se adaptar aos desafios e exigências peculiares da vida acadêmica e construir novos vínculos sociais nesse meio. Essas mudanças são desafiantes e possuem elevado potencial estressogênico e, caso o estudante não se ajuste a elas, podem resultar em sofrimento psíquico que, aliado a outros fatores, pode contribuir para a gênese de pensamentos suicidas. ${ }^{3,8}$

A ideação suicida é um comportamento subjetivo associado a uma miríade de causas subjacentes que são complexas de se entender e mensurar e que variam entre diferentes grupos populacionais e etários. Entre universitários, embora escassos, estudos têm sido realizados objetivando identificar a prevalência da ideação suicida, assim como seus fatores associados. ${ }^{5-8}$

Evidências, nesse sentido, têm demonstrado que diferentes aspectos comportamentais e de caráter subjetivo estão relacionados ao comportamento suicida $^{5,8-10}$, destacando-se entre eles a impulsividade. ${ }^{9-10}$ Conceito polissêmico, de maneira geral, a impulsividade pode ser compreendida como uma reação rápida e não planejada adotada sem avaliar todas as suas potenciais implicações e as possíveis consequências, focando-se apenas nas respostas imediatistas, e não nas de longo prazo. $^{10} \mathrm{O}$ comportamento impulsivo impacta negativamente diversas facetas da vida humana com potencial para gerar adoecimento mental, sofrimento psíquico e prejuízos à qualidade de vida. ${ }^{9-11}$ Entre estudantes universitários, a impulsividade tem sido apontada como um fator associado à ideação suicida nessa população. ${ }^{10,11}$

No contexto nacional, são escassos os estudos que avaliam os riscos de comportamentos suicidas entre estudantes universitários e, mais raras ainda, investigações acerca da associação entre impulsividade e ideação suicida nessa população, o que reforça a necessidade de se examinar como esses fenômenos impactam na vida do estudante de graduação. Desse modo, a presente 
pesquisa possui como objetivo avaliar a associação entre impulsividade e ideação suicida em estudantes universitários.

Ressalta-se que este estudo compõe parte de um projeto matricial intitulado "Ideação suicida e fatores associados em estudantes universitários" ${ }^{\prime 12}$, tendo como objetivo principal avaliar a associação entre a impulsividade e a presença da ideação suicida em uma amostra de estudantes de uma universidade pública federal, haja vista que entender essa dinâmica associativa, além de possibilitar uma melhor compreensão dos eventos, permite traçar um diagnóstico situacional e a implementação de medidas preventivas, baseadas em evidências, por parte das instituições de ensino superior do país.

\section{MÉTODO}

Realizou-se estudo analítico, de delineamento transversal, com estudantes universitários da Universidade Federal de Mato Grosso (UFMT), campus Cuiabá-MT. A amostra para esta pesquisa foi composta por 567 estudantes da graduação, com idade igual ou superior a 18 anos e que responderam adequadamente aos instrumentos de coleta desta investigação. Vale apontar que a amostra total no estudo matricial foi estimada em 714 estudantes de graduação, considerando nível de confiança de $95 \%$, proporção de $50 \%$ e erro amostral de $3,5 \%$ Para essa análise, desconsideraram-se 147 formulários que apresentaram inconsistências, respostas em branco ou que não permitiram a correta avaliação da impulsividade e/ou ideação suicida. Adotou-se método de amostragem probabilística por conglomerados (turmas como unidade amostral) e estratificada (por áreas do conhecimento) a fim de ter uma amostra que representasse adequadamente toda a população do estudo. Das 373 turmas ofertadas no período de estudo, 46 foram aleatoriamente sorteadas por meio do programa MINITAB.

A coleta de dados ocorreu no período de abril e maio de 2015 e foi realizada por aplicadores previamente treinados. Houve distribuição nas salas de aula de instrumentos autoaplicáveis e respondidos de maneira autônoma pelos sujeitos de pesquisa, sem a presença do professor e após breve explicação sobre os objetivos do estudo. Os instrumentos, após o preenchimento, foram devolvidos em urnas dispostas em frente das salas de aula.

Visando caracterizar a amostra, utilizou-se um instrumento fechado e construído pelo pesquisador principal do estudo matricial composto por variáveis sociodemográficas e econômicas. A prevalência de ideação suicida foi aferida por meio da seguinte pergunta dicotômica: "Nos últimos 30 dias você pensou em se matar?", podendo o respondente optar entre 
"sim" ou "não". Vale destacar que esse mesmo questionamento foi utilizado em estudos prévios que avaliaram ideação suicida em amostras com estudantes universitários.

A avaliação da variável impulsividade foi realizada por meio da Escala de Impulsividade de Barratt (BIS11), traduzida e validada para uso no contexto brasileiro. ${ }^{13}$ Trata-se de um instrumento de autopreenchimento, composto por 30 itens relacionados às manifestações da impulsividade, sendo esta dividida em três subdomínios: impulsividade motora, relacionada a não inibição de respostas incoerentes com o contexto, ou seja, tendência a agir sem premeditação; impulsividade atencional, relacionada à tomada de decisão rápida, ou seja, o indivíduo tem tendência de reduzir a atenção sustentada em um estímulo-alvo por intromissão de pensamentos, o que pode levar a apresentar comportamentos descontextualizados por dificuldades em sustentar a atenção; e impulsividade por falta de planejamento, que engloba comportamentos orientados para o presente sem pensar nas consequências da tomada de decisão em longo prazo. ${ }^{13}$

O participante do estudo analisava cada um dos itens considerando seu próprio comportamento e classificava de acordo com uma escala do tipo Likert de quatro pontos, variando de 1 (raramente ou nunca) a 4 (quase sempre/sempre). $\mathrm{O}$ escore geral da escala varia de 30 a 120 pontos, sendo classificados os indivíduos com pontuação $<52$ como muito controlados, de 52-71 pontos com limites normais de impulsividade e $\geq 72$ como altamente impulsivos. $^{13}$

Além de um escore global, a BIS-11 permite o cálculo de escores parciais referentes aos seus três subdomínios da impulsividade: motora (itens 2, 3, 4, 16, 17, 19, 21, 22, 23, 25 e 30), atencional (itens 6, $5,9,11,20,24,26,28)$ e por não planejamento (itens $1,7,8,10,12,13,14$, $15,18,27,29) .^{13}$

Os dados foram digitados no programa Microsoft Office Excel for Windows ${ }^{\circledR}$, com dupla digitação, visando ao pareamento posterior por meio dos recursos do EpiInfo, versão 7.0. Esse procedimento garantiu a identificação de possíveis inconsistências entre as digitações e correção antes da etapa analítica. Após a análise individual das variáveis do estudo, utilizando elementos da estatística descritiva básica, os resultados obtidos foram tratados de maneira associativa por meio do programa Statistical Package for the Social Sciences (SPSS), versão 17.0, verificando-se quais elementos sociodemográficos, econômicos e da impulsividade se associaram de maneira estatisticamente significativa com a ideação suicida. As associações foram verificadas 
pelo teste do qui-quadrado de Pearson (ou o teste exato de Fisher, quando pertinente), adotando-se nível de significância de 5\% e intervalo de confiança (IC) de $95 \%$.

A pesquisa matricial foi submetida à análise do Comitê de Ética em Pesquisa da UFMT em cumprimento aos princípios éticos determinados pela Resolução $\mathrm{n}^{\circ} 510$, de abril de 2016, do Conselho Nacional de Saúde (CNS), sendo aprovada sob parecer $\mathrm{n}^{\circ}$ 1.021.217. Na ocasião da coleta, os participantes foram informados quanto ao direito de se recusarem a participar e todos os procedimentos para garantia de confidencialidade, anonimato, privacidade e não maleficência foram adotados. ${ }^{14}$

\section{RESULTADOS}

A amostra de estudantes universitários caracterizou-se pela predominância de sujeitos do sexo feminino (52,7\%), na faixa etária de 18 a 24 anos $(68,9 \%)$, de cor parda $(42,3 \%)$, que não trabalhavam $(62,2 \%)$ e com impulsividade classificada dentro dos limites de normalidade, segundo a BIS-11 $(65,96 \%)$. Quanto à prevalência de ideação suicida entre os estudantes universitários, dos 567 questionários validados, $9,7 \%$ destes relataram ideação nos últimos 30 dias (Tabela 1).

Tabela 1 - Caracterização sociodemográfica, de impulsividade e presença de ideação suicida entre os estudantes universitários da UFMT. Cuiabá, MT, Brasil, 2015

\begin{tabular}{llll}
\hline Variáveis & $\mathrm{n}(567)$ & $(\%)$ & $\mathrm{IC} 95 \%$ \\
\hline Idade & & & \\
18 a 24 anos & 391 & 68,96 & $(65,03 ; 72,63)$ \\
25 a 32 anos & 91 & 16,05 & $(13,26 ; 19,30)$ \\
32 anos ou mais & 85 & 14,99 & $(12,29 ; 18,16)$ \\
Sexo & & & \\
Feminino & 299 & 52,73 & $(48,62 ; 56,81)$ \\
$\quad$ Masculino & 268 & 47,27 & $(43,19 ; 51,38)$ \\
Cor & & & \\
Negra & 91 & 16,05 & $(13,26 ; 19,30)$ \\
Parda & 240 & 42,33 & $(38,33 ; 46,43)$ \\
Branca & 236 & 41,62 & $(37,63 ; 45,72)$ \\
Situação Profissional & & & \\
$\quad$ Trabalha & 214 & 37,74 & $(33,85 ; 41,80)$ \\
$\quad$ Não trabalha & 353 & 62,26 & $(58,20 ; 66,15)$ \\
Mora sozinho & & & \\
Sim & 89 & 15,70 & $(12,93 ; 18,92)$ \\
Não & 478 & 84,30 & $(81,08 ; 87,07)$ \\
Impulsividade & & & \\
Muito controlado & 114 & 20,11 & $(17,01 ; 23,60)$ \\
Normal & 374 & 65,96 & $(61,97 ; 69,74)$ \\
Altamente impulsivo & 79 & 13,93 & $(11,32 ; 17,03)$ \\
Ideação suicida nos últimos trintas dias? & & & \\
Sim & 55 & 9,70 & $(7,53 ; 12,41)$ \\
$\quad$ Não & 512 & 90,30 & $(87,59 ; 92,47)$ \\
Fonte: elaborado pelos autores Nota. IC= Intervalo de Confiança. & & &
\end{tabular}


Dados não apresentados, porém evidenciados no estudo, demonstraram que os indivíduos que apresentaram ideação suicida em sua maioria eram do sexo feminino $(63,64 \%)$, estavam na faixa etária entre 18 e 24 anos (74,55\%), eram de raça branca $(50,91 \%)$, não trabalhavam no momento da coleta de dados $(67,27 \%)$ e, majoritariamente, não moravam sozinhos $(78,18 \%)$.
A Tabela 2 apresenta os escores geral e dos subdomínios de impulsividade avaliados pela BIS-11. Observa-se média de pontuação total dos itens da escala de 60,38 pontos, condizentes com indicadores de impulsividade considerados normais. Os valores médios para os subdomínios foram, respectivamente, $\quad 19,10 \quad$ pontos (impulsividade motora), 17,10 pontos (impulsividade atencional) e 24,19 pontos (impulsividade por falta de planejamento).

Tabela 2 - Escala de impulsividade de Barrat BIS 11 - pontuação geral e subdomínios dos estudantes universitários da UFMT. Cuiabá, MT, Brasil, 2015

\begin{tabular}{lll}
\hline BIS 11 - Subdomínios & Média (DP) & Mín. - Máx. \\
\hline Motor & $19,10 \pm 4,058$ & $11,000-35,000$ \\
Atencional & $17,10 \pm 4,153$ & $8,000-31,000$ \\
Falta de planejamento & $24,19 \pm 4,594$ & $12,000-39,000$ \\
Geral & $60,38 \pm 10,612$ & $34,000-105,000$
\end{tabular}

Fonte: elaborado pelos autores Nota. DP = Desvio Padrão.

A Tabela 3 apresenta a associação das variáveis sociodemográficas dos estudantes universitários da UFMT com a presença da ideação suicida. Quanto à impulsividade, constata-se que a ideação suicida foi significativamente associada entre os categorizados como altamente controlados e os altamente impulsivos $(\mathrm{p}<0,001)$, respectivamente. 
Tabela 3 - Associação entre as variáveis demográficas, sociais e de impulsividade com a presença da ideação suicida nos últimos 30 dias em estudantes universitários da UFMT. Cuiabá, MT, Brasil, 2015

\begin{tabular}{|c|c|c|c|c|c|}
\hline \multirow[t]{2}{*}{ Variáveis } & \multicolumn{4}{|c|}{$\begin{array}{l}\text { Ideação suicida } \\
\text { Sim }\end{array}$} & \multirow[t]{2}{*}{ p-valor } \\
\hline & $\mathrm{N}$ & $(\%)$ & $\mathrm{N}$ & $(\%)$ & \\
\hline \multicolumn{6}{|l|}{ Idade } \\
\hline 18 a 24 anos & 41 & $(10,49)$ & 350 & $(89,51)$ & .346 \\
\hline 25 a 31 anos & 10 & $(10,99)$ & 81 & $(89,01)$ & .650 \\
\hline 32 anos ou mais & 4 & $(4,71)$ & 81 & $(95,29)$ & - \\
\hline \multicolumn{6}{|l|}{ Sexo } \\
\hline Feminino & 35 & $(11,71)$ & 264 & $(88,29)$ & .088 \\
\hline Masculino & 20 & $(7,46)$ & 248 & $(92,54)$ & - \\
\hline \multicolumn{6}{|l|}{ Cor } \\
\hline Negro & 9 & $(9,89)$ & 82 & $(90,11)$ & .947 \\
\hline Pardo & 18 & $(7,50)$ & 222 & $(92,50)$ & .129 \\
\hline Branco & 28 & $(11,86)$ & 208 & $(88,14)$ & - \\
\hline \multicolumn{6}{|l|}{ Situação Profissional } \\
\hline Trabalha & 18 & $(8,41)$ & 196 & $(91,59)$ & .419 \\
\hline Não trabalha & 37 & $(10,48)$ & 316 & $(89,52)$ & - \\
\hline \multicolumn{6}{|l|}{ Mora sozinho } \\
\hline Sim & 12 & $(13,48)$ & 77 & $(86,52)$ & .189 \\
\hline Não & 43 & $(9,00)$ & 435 & $(91,00)$ & - \\
\hline \multicolumn{6}{|l|}{ Impulsividade } \\
\hline Muito controlado & 2 & $(1,75)$ & 112 & $(98,25)$ & $<.001^{\mathrm{EF}}$ \\
\hline Altamente impulsivos & 20 & $(25,32)$ & 59 & $(74,68)$ & $<.001$ \\
\hline Normal & 33 & $(8,82)$ & 341 & $(91,18)$ & - \\
\hline
\end{tabular}

\section{DISCUSSÃO}

Este estudo teve como objetivo avaliar a associação entre a impulsividade e a presença da ideação suicida em estudantes da Universidade Federal de Mato Grosso Campus Cuiabá, no qual as características evidenciadas assemelham-se aos resultados encontrados em investigações prévias sobre a ideação suicida nessa mesma população, destacando-se a prevalência no sexo feminino $^{7}$, na faixa etária compreendida entre 18 e 24 anos, na raça branca ${ }^{15}$, sem vínculo empregatício ${ }^{16} \mathrm{e}$ que não moravam sozinhos. ${ }^{7}$
A prevalência encontrada nesta pesquisa para a ideação suicida é semelhante à obtida em estudos com universitários de outros países. Um deles, realizado em uma instituição de ensino superior de Portugal $(\mathrm{n}=1.074)$, apontou prevalência de ideação suicida de 7,8\% outro, realizado com 5.245 alunos na China, encontrou $9,1 \%$ dos estudantes com pensamentos suicidas. ${ }^{17}$

Ressalta-se aqui a escassez de informações sobre a presença de ideação em jovens universitários brasileiros, fato este que tem dificultado comparações com esse 
segmento populacional. No Brasil, estudo realizado com universitários $(\mathrm{n}=637)$, nas cidades de Natal-RN e João Pessoa-PB, no período de 2007 e 2008, encontrou indicadores alarmantes de prevalência de ideação suicida em sua amostra $(52,45 \%) .^{5}$ Mais recentemente, estudo que investigou fatores associados à ideação suicida em universitários da área da saúde $(\mathrm{n}=142)$ identificou que $22 \%$ dos estudantes apresentavam pensamentos suicidas. ${ }^{8}$

Chamam atenção as altas prevalências de ideação suicida obtidas nos estudos brasileiros ${ }^{5,8}$, achados discrepantes em relação aos resultados de estudos realizados com outros jovens, bem como na presente pesquisa. No entanto, tais diferenças podem ter ocorrido perante as diversidades regionais, diferenças do grupo populacional e, ainda, em decorrência dos métodos e instrumentos utilizados. Outra possibilidade pode dizer respeito aos diferentes tamanhos de amostras utilizadas nos estudos com essa população. ${ }^{17}$

Em relação à impulsividade, 65,96\% dos universitários encontravam-se dentro dos limites normais e $13,93 \%$ eram altamente impulsivos. Tais achados vão ao encontro do estudo desenvolvido com universitários no Sul do Brasil $(\mathrm{n}=129)$, com prevalência de 69\% para limites normais e $31 \%$ para os indivíduos altamente impulsivos. ${ }^{10}$
A impulsividade é uma predisposição para rápidas e não planejadas reações oriundas de estímulos internos ou externos que não levam em consideração os resultados negativos que podem surgir. ${ }^{9,10}$ Seu aumento pode levar a um comportamento agressivo, violento, não planejado, associado à percepção de ameaça ou frustração imediata, às emoções negativas como medo e raiva, e caracterizado por altos níveis de ativação do sistema nervoso autônomo. A agressão impulsiva ou reativa pode ser considerada como uma reação normal e desejável às ameaças ambientais. Porém, pode ser patológica quando sua intensidade é desproporcional ou dirigida ao alvo errado, gerando consequências negativas.9-11,24

Por mais que não exista um consenso entre os pesquisadores quanto a sua definição e embora existam diferentes conceitos, o ponto comum entre os autores se refere a sua multidimensionalidade. ${ }^{18}$ Dessa forma, sua avaliação pode se dar por meio de instrumentos que investiguem domínios específicos, por exemplo, a Escala de Impulsividade de Barrat, que tem sido amplamente descrita na literatura como uma das melhores medidas de autorrelato estabelecida de impulsividade. ${ }^{13}$

Com o uso desse instrumento, no presente estudo, os achados foram classificados em domínios: motor, atencional e de falta de planejamento, e os 
resultados encontrados são discretamente inferiores quando comparados ao estudo realizado com universitários do Sul do Brasil, no qual foram encontradas médias de 23,87 para o escore motor, 22,43 para o atencional e 30,5 para a falta de planejamento. ${ }^{10} \mathrm{Em}$ ambos os estudos os valores mais altos foram obtidos no domínio falta de planejamento.

Hipóteses sugerem que as pessoas incapazes de utilizar as experiências emocionais e motivacionais passadas para guiar as decisões atuais apresentam maiores escores de impulsividade para a falta de planejamento. ${ }^{18} \mathrm{Se}$ a falta de planejamento envolve comportamentos do presente sem pensar nas consequências em longo prazo, a identificação da presença da ideação suicida nesses indivíduos impulsivos é fundamental, visto que podem evoluir da ideação para a ação impensada, ou seja, a tentativa de suicídio, culminando em morte. $^{10}$

A associação encontrada no presente estudo entre impulsividade e ideação suicida também foi demonstrada em outros estudos como o desenvolvido com 258 estudantes universitários na Colômbia, que identificou associação significativa $(p<0,001)$ entre as variáveis. ${ }^{6}$ Em Portugal, uma pesquisa com 1.074 universitários objetivando avaliar a ideação suicida e sua relação com algumas variáveis psicossociais também comprovou associação estatística entre a ideação suicida e a impulsividade $(\mathrm{p}<0,001)$, afirmando que quanto menor a impulsividade, menor o risco de ideação suicida. $^{7}$

Estudo de caso-controle, realizado na China, analisou 392 casos de suicídio na população com idade de 15 a 34 anos com o objetivo de explorar os efeitos da impulsividade sobre os suicídios chineses e o papel que esta desempenha na relação entre eventos negativos da vida $\mathrm{e}$ comportamento suicida. Os resultados apontaram a impulsividade como um importante preditor de suicídios naquela população. ${ }^{19}$ Nessa perspectiva, tem se considerado que a impulsividade tem emergido como um promissor marcador de risco para o suicídio.

A impulsividade quando está associada à presença de ideação suicida tornaria o indivíduo mais predisposto a cometer o suicídio. O comportamento suicida, em geral, perpassa linear e continuamente as etapas de ideação, planejamento, tentativas e suicídio, permitindo que a investigação de pensamentos suicidas constitua uma oportunidade precoce para intervenção e prevenção. $^{1,3}$ Entretanto, essa sequência clássica de comportamentos pode não ser aplicada para indivíduos impulsivos que podem tirar a própria vida sem 
planejamento e/ou longos períodos de ideação.

Ressalta-se que a associação obtida no presente estudo entre alta impulsividade e ideação suicida vai ao encontro da literatura, conforme discutido acima. No entanto, a associação entre os estudantes altamente controlados quanto à impulsividade e ideação precisa ser melhor investigada, considerando que tal achado não foi descrito em outros estudos com universitários. Vale salientar que para as demais variáveis investigadas nenhuma associação significativa foi observada.

Sendo a literatura produzida sobre essa temática ainda consideravelmente incipiente no Brasil, uma das limitações do presente estudo refere-se à dificuldade de comparação dos achados com populações semelhantes, o que possibilitaria levantar hipóteses para o desenvolvimento de novos estudos visando diminuir as lacunas científicas. Outro aspecto trata do delineamento transversal do estudo que não permite estabelecer relações de causa e efeito. No entanto, os achados sugerem que tais aspectos possam ser incorporados na elaboração de medidas preventivas nas instituições de ensino superior que devem oferecer programas que promovam a saúde mental em ambientes acadêmicos.

\section{CONCLUSÃO}

Neste estudo, verificou-se que a prevalência de ideação suicida entre estudantes universitários é semelhante à descrita em estudos internacionais, porém consideravelmente inferior ao único estudo existente no Brasil com essa população. Quanto à impulsividade, a maioria manteve-se dentro dos padrões normais, contudo os estudantes altamente impulsivos e muito controlados apresentaram associação significativa com a presença da ideação suicida. Achado que precisa ser melhor investigado em pesquisas posteriores.

Os dados obtidos contribuem para a produção de conhecimento sobre a temática de forma substancial dada a escassez de informações existentes. Dessa forma, apesar das limitações impostas pelo tipo de estudo (transversal) e da ausência de pesquisas que investiguem o mesmo objeto, o que dificulta a comparação dos dados, os achados descritos poderão contribuir substancialmente para o avanço do conhecimento, atualização de profissionais e demais pessoas ligadas à área de saúde mental, assim como repercutir na ampliação da discussão da temática e consequentemente melhoria da assistência a essa população. 


\section{REFERÊNCIAS}

1. Teixeira SMO, Souza LEC, Viana LMM. O suicídio como questão de saúde pública. Rev Bras Promoç Saúde [Internet]. 2018 [citado em $10 \mathrm{dez}$ 2019]; 31(3):1-3. doi: http://dx.doi.org/10.5020/18061230.20 18.8565. Disponível em: https://periodicos.unifor.br/RBPS/articl e/view/8565

2. World Health Organization. Preventing suicide: a global imperative. Geneva: WHO; 2014.

3. Botega NJ. Crise suicida: avaliação e manejo. Porto Alegre: Artmed; 2015.

4. Faure MI, Urquidi C, Bustamante F, Florenzano R, Ampuero K, Terán L et al. Asociación entre la calidad de vida relacionada con la salud y riesgo suicida en adolescentes: estudio transversal. Rev Chil Pediatr. [Internet]. 2018 [citado em 28 out 2018]; 89(3):318-24. Disponível em: https://www.scielo.cl/pdf/rcp/v89n3/03 70-4106-rcp-00103.pdf. doi: http://dx.doi.org/10.4067/S037041062018005000103

5. Dutra E. Suicídio de universitários: o vazio existencial de jovens na contemporaneidade. Estud Pesqui Psicol. [Internet]. 2012 [citado em 05 out 2018]; 12(3):924-37. Disponíevel em: https://www.epublicacoes.uerj.br/index.php/revispsi/ article/view/8229/5986. doi: https://doi.org/10.12957/epp.2012.8229

6. Siabato Macías, Salamanca Camargo Y. Factores asociados a ideación suicida em universitários. Psychol Av Discip. [Internet]. 2015 [citado em 05 out 2018]; 9(1):71-81. Disponível em: https://revistas.usb.edu.co/index.php/Ps ychologia/article/view/994/733. doi: https://doi.org/10.21500/19002386.994

7. Gonçalves AM, Sequeira CAC, Duarte JC, Freitas PP de. Suicidal ideation on higher education students: influence of some psychosocial variables. Arch Psychiatr Nurs. [Internet]. 2016 [citado em 17 out 2018]; 30(2):162-66. doi: https://doi.org/10.1016/j.apnu.2015.08. 005

8. Veloso LUP, Lima CLS, Sales JCS, Monteiro CFS, Gonçalves MAS, Silva Júnior FJG. Ideação suicida em universitários da área da saúde: prevalência e fatores associados. Rev Gaúch Enferm. [Internet]. 2019 [citado em 10 jan 2020]; 40:e20180144.

Disponível em:

https://www.scielo.br/j/rgenf/a/JttXRN sGZJGqtG3b4NnBZHS/?lang=pt. doi:https://doi.org/10.1590/19831447.2019.20180144

9. Hadzic A, Spangenberg L, Hallensleben N, Forkmann T, Rath D, Straub A, et al. The association of traitimpulsivity and suicidal ideation and its fluctuation in the context of the Interpersonal Theory of Suicide. Compr Psychiatry [Internet]. 2019 [citado em 10 jan 2020]; 98:152158. Disponível em:

https://www.sciencedirect.com/science/ article/pii/S0010440X19300811. doi: https://doi.org/10.1016/j.comppsych.20 19.152158

10. Parcias SR, Sombrio LS, Flügel NT, Rosario MJP do, Souza MC, Guimarães ACA. Comportamento impulsivo: estudo em uma população de universitários. Rev Atenção Saude [Internet]. 2014 [citado em 24 out 2018]; 12(42):36-41. Disponível em: https://seer.uscs.edu.br/index.php/revist a_ciencias_saude/article/view/2176/16 54.... doi: 10.13037/rbcs.vol12n42.2176

11. Loftis MA, Michael T, Luke C. College student suicide risk: the relationship between alexithymia, impulsivity, and internal locus of control. Int J Educ Psychol. [Internet]. 2019 [citado em 10 jan 2020]; 8(3):246-69. Disponível em: https://hipatiapress.com/hpjournals/ind ex.php/ijep/article/view/3991. doi: https://doi.org/10.17583/ijep.2019.399 1 
12. Santos HGB, Marcon SR, Espinosa MM, Baptista MN, Paulo PMC. Fatores associados à presença de ideação suicida entre universitários. Rev Latinoam Enferm. [Internet]. 2017 [citado em 23 out 2018]; 25:e2878. Disponível em: https://www.scielo.br/j/rlae/a/PhmjVpP 5Z86X8vkHgCdqz9D/?lang=pt\&forma $\mathrm{t}=$ pdf. doi: https://doi.org/10.1590/15188345.1592.2878

13. Malloy-Diniz LF, Mattos P, Leite WB, Abreu N, Coutinho G, de Paula JJ et al. Translation and cultural adaptation of Barratt Impulsiveness Scale (BIS-11) for administration in Brazilian adults. J Bras Psiquiatr. [Internet]. 2010 [citado em 25 out 2018]; 59(2):99-105.

Disponível em:

https://www.scielo.br/j/jbpsiq/a/KJHvd cGNxgqy4DGCjHBnxqM/?lang=pt. doi: https://doi.org/10.1590/S004720852010000200004

14. Conselho Nacional de Saúde (Brasil). Resolução ${ }^{\circ} 510$, de 7 de abril de 2016. Aprova normas regulamentadoras de pesquisas envolvendo seres humanos [Internet]. D.O.U., Brasília, DF, 24 maio 2016 [citado em 22 set 2021]; seção I, (98):44-46. Disponível em: http://conselho.saude.gov.br/resolucoes /2016/Reso510.pdf

15. Pires MCC, Raposo MCF, Sougey EB, Bastos Filho OC, Silva TS, Passos MP. Indicadores de risco para tentativa de suicídio por envenenamento: um estudo caso-controle. J Bras Psiquiatr. [Internet]. 2015 [citado em 17 out 2018]; 64(3):193-9. Disponível em: https://www.scielo.br/j/jbpsiq/a/rKgyjd D6rwWxXJBcqC8kxHp/?lang=pt. doi: https://doi.org/10.1590/00472085000000078

16. Lee GY, Choi YJ. Association of school, family, and mental health characteristics with suicidal ideation among Korean adolescents. Res Nurs Health. [Internet]. 2015 [citado em 17 out 2018]; 38(4):301-10. doi: https://doi.org/10.1002/nur.21661

17. Wang L, He CZ, Yu YM, Qiu XH, Yang XX, Qiao ZX et al. Associations between impulsivity, aggression, and suicide in Chinese college students. BMC Public Health [Internet]. 2014 [citado em 17 out 2018]; 14(1):551. Disponível em: https://bmcpublichealth.biomedcentral. com/track/pdf/10.1186/1471-2458-14551.pdf. doi:10.1186/1471-2458-14551

18. Creswell KG, Wright AGC, Flory JD, Skrzynski CJ, Manuck SB.

Multidimensional assessment of impulsity-related measures in relation to externalizing behaviors. Psychol. Med. [Internet]. 2019 [citado em 10 jan 2020]; 49(10):1678-90. Disponível em: https://www.ncbi.nlm.nih.gov/pmc/arti cles/PMC7043188/pdf/nihms1558890.pdf. doi: https://doi.org/10.1017/S00332917180 0229

19. Zhang J, Lin L. The moderating effects of impulsivity on Chinese rural young suicide. J Clin Psychol. [Internet]. 2014 Jun [citado em 3 out 2018]; 70(6):57988. doi: 10.1002/jclp.22039

RECEBIDO: 07/02/2020

APROVADO: 04/12/2020 PUBLICADO: 09/2021 